\title{
MATHEMATICAL MODELING AND OPTIMAL CONTROL OF EBOLA VIRUS TRANSMISSION DYNAMICS
}

\author{
Amira Rachah ${ }^{1}$, Marwen Saidi ${ }^{2}$ \\ ${ }^{1}$ SINTEF Industry, Postboks 4760 Torgarden \\ Trondheim - 7465, NORWAY \\ ${ }^{2}$ INRA Versailles-Grignon \\ Versailles - 78026 Cedex, FRANCE
}

\begin{abstract}
This paper investigates a dynamic model for assessing the populationlevel impact of isolation of infectious individuals during the 2015-outbreak of Ebola virus in Liberia. The model includes demographic effects, latent undetectable and latent detectable individuals. The paper presents different optimal control strategies associated with isolating infectious symptomatic individuals in order to control the propagation of the virus.
\end{abstract}

AMS Subject Classification: 49K15, 92D25, 92D30

Key Words: mathematical modeling; optimal control; Ebola; isolation

\section{Introduction}

Ebola virus disease is one of the most virulent viral diseases of humans, with a case fatality ratio estimated between 25 and $90 \%$ [1]. It is named after the Ebola River in the Democratic Republic of the Congo, where it was firstly discovered in 1976 [2, 3, 4]. Recently, the virus is identified in West Africa mainly in Liberia, Sierra Leone and Guinea $[2,5]$. The virus is transmitted initially to human by contact with an infected animal's body fluid. It is most commonly spread by contact with blood and secretions, either via direct contact

Received: December 4, 2020

(C) 2021 Academic Publications

${ }^{\S}$ Correspondence author 
(through broken skin or mucous membranes in, for example, the eyes, nose, or mouth) with the infected individual or fluids on clothing or other surfaces, as well as needles $[3,6,7,8]$. It is previously known as Ebola hemorrhagic fever. Early symptoms of Ebola include: fever, headache, joint and muscle aches, sore throat, and weakness. Later symptoms include diarrhea, vomiting, stomach pain, hiccups, rashes, bleeding, and organ failure. When Ebola progresses to external and internal bleeding, it is almost always fatal $[9,10,11]$. The 2015 outbreak in West Africa has caused more than 28000 infections and 11100 deaths by December 2015. The epidemic continued increasing due to socioeconomic disadvantage and shortages in the health systems of the three mainly affected countries Liberia, Sierra Leone and Guinea [4]. The survival of the virus in the environment, due to poor hygienic and sanitary conditions, is probably another source of Ebola infection in these countries [12].

Mathematical models of infectious disease transmission dynamics play an important role in helping to quantify possible infectious disease control and mitigation strategies. Modeling and optimal control theory have a powerful tool for investigating human infectious diseases, contributing to the understanding of the dynamics of diseases, providing useful predictions about the potential transmission of the virus and the effectiveness of possible control measures which can provide valuable information for public health policy makers. Numerous mathematical modeling studies have been conducted to study the transmission dynamics and control of infectious diseases using compartmental models $[13,14]$. The inclusion, in an epidemic model, of some practical control strategies (vaccines, treatment, educational campaigns) provides a rational basis for policies designed to control the spread of the virus [15, 16, 17, 18, 19]. Several models have been used to investigate how to control more effectively emerging and re-emerging infectious disease such as smallpox, HIV (Human Immunodeficiency Virus), influenza and SARS (Severe Acute Respiratory Syndrome) using isolation $[20,21,22,23,24]$. The mainly aim of public health policy is to decrease these burdens by reducing transmission or metigating severity. Isolation strategy is one of the most basic public health measure that has historically been used to combat the spread of human infectious diseases. In this study, isolation refers to the removal of latent detectable and infectious symptomatic individuals from the general population. The use of isolation as primary control strategy presents significant logistical and economic strain on a public health system's resources.

The purpose of the current study is to use an advanced compartmental model for realistically assessing the population-level impact of isolation on the spread and control of the 2015 Ebola virus outbreak in Liberia. The paper is 
organized as follows. In Section 2 we present the formulation and the basic analysis of the model that describes the dynamics of Ebola virus by including demographic effects, latent undetectable and latent detectable compartments with isolation of infectious individuals. A numerical simulations of full model, in which we use vital dynamics parameters of Liberia estimated in 2015 is presented in Section 3. Then, we use the obtained model to discuss, in Section 4, different control strategies of the propagation of the virus. We end with Section 5 of conclusions.

\section{Formulation and analysis of the model}

The model is an improvement of the SEIR model in which the total population is split into four mutually exclusive epidemiological classes: Susceptible compartment $S(t)$ which denotes individuals who are susceptible to catch the virus and so might become infectious if exposed, Exposed compartment $E(t)$ which denotes the individuals who are infected but the symptoms of the virus are not yet visible, Infectious compartment $I(t)$ which denotes infectious individuals who are suffering the symptoms of Ebola and able to spread the virus through contact with susceptible classes of individuals, and Recovered compartment $R(t)$ which denotes individuals who have immune to the infection. As an improvement of the SEIR model, we base our study on a deteministic ordinary differential equations epidemic model in which the total population $N$ is divided into six mutually exclusive epidemiological classes: Susceptible individuals $S$, latent undetectable individuals $E_{1}$, latent detectable individuals $E_{2}$, infectious symptomatic individuals $I$, isolated individuals $J$, and individuals removed from isolation after recovery $R(t)$ which denotes the removed compartment. In this model, scueptible individuals become infected and latent through contacy with infectious individual at the rate $\frac{\beta(I+k J)}{N}$, where $\beta$ is the mean transmission rate per day, and $k$ denotes the relative transmissibility of isolated individuals to measure the effectiveness of isolation of infectious individuals. Latent undetectable individuals $E_{1}$ enter the latent detectable group $E_{2}$ at rate $\sigma_{1}$, and become infectious symptomatic at rate $\sigma_{2}$. We assume that the latent detectable group represent individuals with a viral load above the detection limit of the specific diagnostic test. Infectious individuals are isolated at the rate $\theta$ where Recovered individuals are removed from isolation after recovery at the rate $\gamma$. Furthermore, each group decreases at the natural death rate $\mu$ where the susceptible group increases at the natural birth rate. A schematic representation of the flow of individuals between the different classes is shown 
in Figure 1.

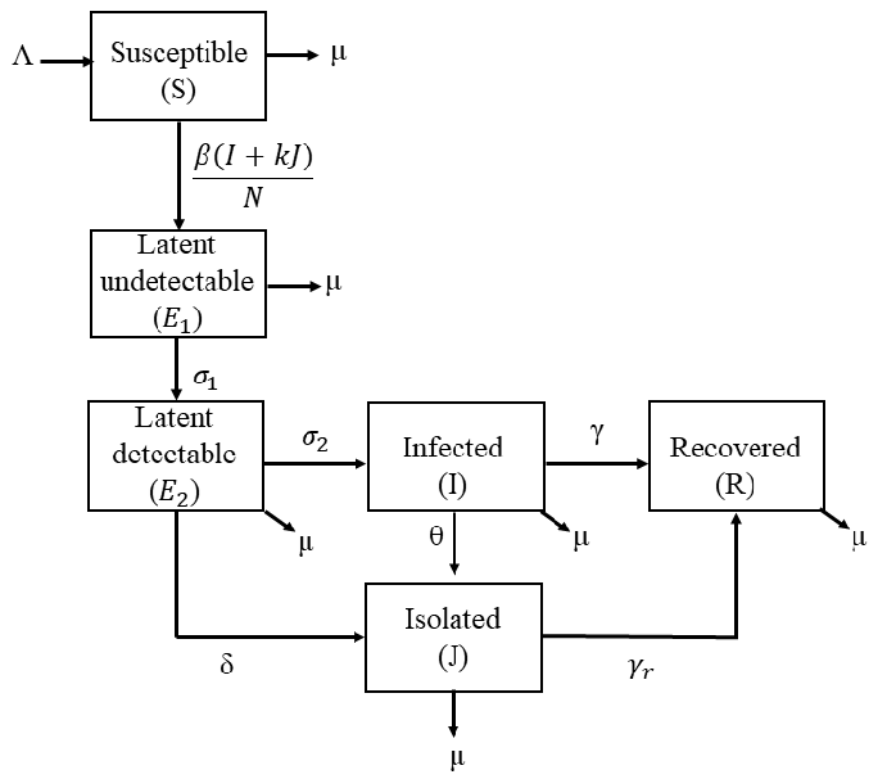

Figure 1: Schematic representation of the flow of individuals between the different classes.

The model is described by the following system of nonlinear ordinary differential equations:

$$
\begin{aligned}
& \frac{d S(t)}{d t}=\Lambda-\beta S(t)\left(\frac{I(t)+k J(t)}{N}\right)-\mu S(t), \\
& \frac{d E_{1}(t)}{d t}=\beta S(t)\left(\frac{I(t)+k J(t)}{N}\right)-\left(\sigma_{1}+\mu\right) E_{1}(t), \\
& \frac{d E_{2}(t)}{d t}=\sigma_{1} E_{1}(t)-\left(\sigma_{2}+\delta+\mu\right) E_{2}(t), \\
& \frac{d I(t)}{d t}=\sigma_{2} E_{2}(t)-(\theta+\gamma+\mu) I(t) \\
& \frac{d J(t)}{d t}=\theta I(t)+\delta E_{2}(t)-\left(\gamma_{r}+\mu\right) J(t), \\
& \frac{d R(t)}{d t}=\gamma I(t)+\gamma_{r} J(t)-\mu R(t) .
\end{aligned}
$$

The model is parametrized to the transmission dynamics of Ebola virus in West Africa using published estimates. The commun parameters estimated in 
previous study of Ebola virus in West Africa are: The mean incubation time $\frac{1}{\sigma_{1}}+\frac{1}{\sigma_{2}}$ is equal to 7 days where $\frac{1}{\sigma_{1}}=4$ days and $\frac{1}{\sigma_{1}}=3$ days $[25,26,27]$. The mean infection time is given by $\frac{1}{\theta}=$ days $[28,29,27]$. The description of the rest of commun parameters is given in Table 1.

Table 1: Description of commun parameters of the model.

\begin{tabular}{ccc}
\hline parameters & Description & values \\
\hline$k$ & Effectiveness of isolation of infectious & 0.5 \\
$\frac{1}{\sigma_{1}}$ & Latent undetectable period & 4 days \\
$\frac{1}{\sigma_{2}}$ & Latent detectable period & 3 days \\
$\frac{1}{\sigma_{1}}+\frac{1}{\sigma_{2}}$ & Mean incubation time & 7 days \\
$\frac{1}{\theta}$ & Mean infection time & 5 days \\
$\gamma$ & Rate of recovery fom $I$ to $R$ & 0.1 \\
$\gamma_{r}$ & Rate of recovery fom $E_{2}$ to $R$ & 0.2 \\
$\delta$ & Rate of isolation fom $E_{2}$ to $J$ & 0.16 \\
\hline
\end{tabular}

The trasmission rate is estimated in recent study of Ebola virus occuring in Liberia in 2015, [30]. Furthermore, the 2015 estimation of the total population, the natural birth and death rates are published on the website of Statistiques Mondiales (2015), [31]. The description of the transmission rate, the total population, the natural birth and death rates for Liberia are given in Table 2 .

The basic qualitative property of the model 1 , with respect to the nonnegativity of solutions, will now be explored.

Lemma 1. Suppose that the initial values $S(0), E_{1}(0), E_{2}(0), I(0), J(0)$, $R(0)$ of the model (1) are all nonnegative. Then, the solution of (1) starting with these initial values will remain nonnegative for all time $t>0$.

Proof. The proof for the nonnegativity is by contradiction (see Theorem A4 of [32]). Suppose that the statement of the lemma (with respect to nonnegativity) does not hold. That is, there is at least one of the six state variables of the model (1), and a $t=t^{*} \geq 0$, such that the value of this state variable goes through 0 at $t=t^{*}$, and all state variables of the model take nonnegative values 
for $0 \leq t \leq t^{*}$. For example, consider the state variable is $S(t)$. It can be seen that the derivative of $S(t)$ is positive if $S(t)=0$. Hence, $S(t)$ cannot decrease further once it has reached 0 . The case of each of the other state variable of the model can be shown in a similar way.

Table 2: Demographic effects and ransmission rate in Liberia published on the website of Statistiques Mondiales (2015), [31].

\begin{tabular}{ccc}
\hline parameters & Description & $\begin{array}{c}\text { values } \\
\text { Liberia }\end{array}$ \\
\hline$\Lambda$ & Birth rate & 0.03441 \\
$\mu$ & Death rate & 0.00969 \\
$N$ & Total population & 4195666 \\
$\beta$ & Transmission rate & 0.3906 \\
\hline
\end{tabular}

\section{Numerical simulation}

In this section, we study the impact of the isolation on Ebola virus during the 2015 outbreaks occuring in Liberia. In order to provide useful predictions about the potential transmission of the virus and the effectiveness of isolation, we study the numerical resolution of the model in three scenarios: (1) isolation of latent detectable individuals only; (2) isolation of symptomatic infectious individuals only; (3) isolation of latent detectable and symptomatic infectious individuals. By comparing between the three scenarios, we can see the impact of isolation, in each scenario, on all the classes of individuals. The description of initialization parameters in Liberia [31] is detailed in Table 3.

In order to quantify the impact of isolation and compare between the three scenarios, Figures 2, 3 and 4 show, respectively, the evolution of infectious, isolated and recovered individuals, along time, in the three scenarios. In Figure 2 , the time-dependent curve of infectious symptomatic individuals shows the peak of the curve of infectious symptomatic individuals, which is less important in case of isolation of latent detectable and infectious symptomatic individuals (scenario 3). In fact, the maximum value on the infectious symptomatic curve $I$ in scenario 3 is $2.569 .10^{5}$ individuals means $0.0612 \%$ of the total population, against $2.769 .10^{5}$ means $0.066 \%$ in case of isolation of symptomatic infectious 
Table 3: Description of initialization parameters in Liberia, [31].

\begin{tabular}{ccc}
\hline Computational parameters & Desciption & Values \\
\hline$S(0)$ & $0.8 * N$ & $3.3565 .10^{6}$ \\
$E_{1}(0)$ & $0.05 * N$ & $2.0978 .10^{5}$ \\
$E_{2}(0)$ & $0.1 * N$ & 4.1957 .1005 \\
$I_{(}(0)$ & $0.05 * N$ & $2.0978 .10^{5}$ \\
$J_{(0)}$ & 0 & 0 \\
$R_{(0)}$ & 0 & 0 \\
\hline
\end{tabular}

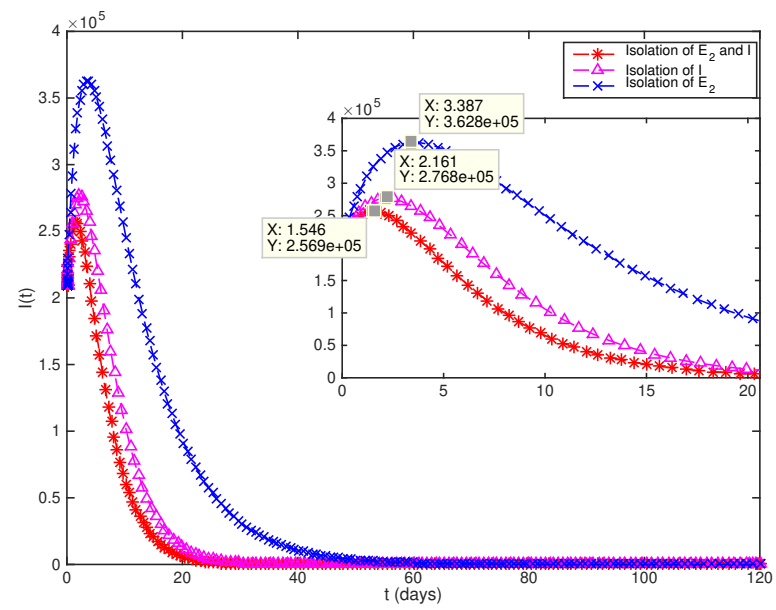

Figure 2: Evolution of infectious individuals $I(t)$. 


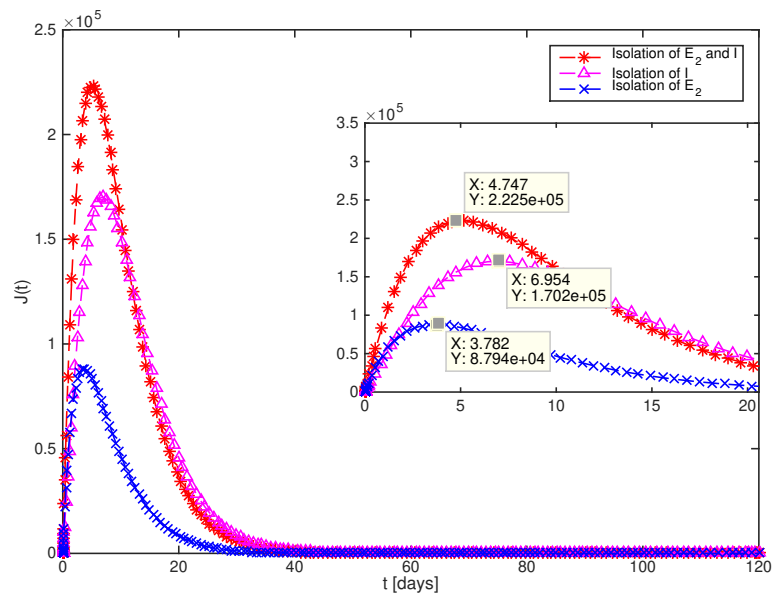

Figure 3: Evolution of isolated individuals $J(t)$.

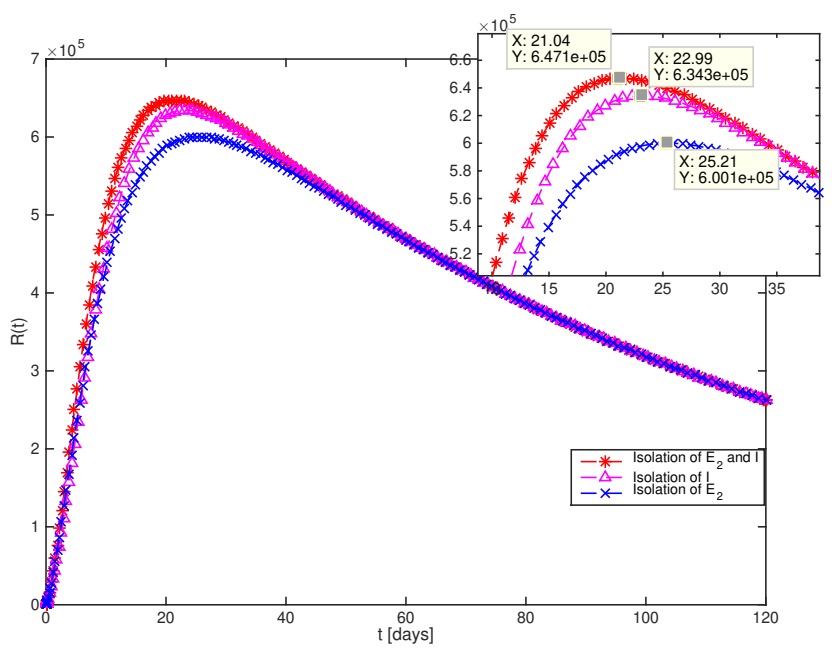

Figure 4: Evolution of recovered individuals $R(t)$. 
only (scenario 2) and $3.628 .10^{5}$ means $0.0865 \%$ in case of isolation of latent detectable only (scenario 3 ). The percentage of symptomatic infectious $0.0612 \%$ of the scenario 3 correspond to the isolation of $2.225 .10^{5}$ individuals $(0.0530 \%$ of the total of population). The percentage of symptomatic infectious $0.066 \%$ of the scenario 2 correspond to the isolation of $1.702 .10^{5}$ individuals $(0.0406 \%$ of the total of population) (see Figure 3). The percentage of symptomatic infectious $0.0865 \%$ of the scenario 1 correspond to the isolation of $8.794 .10^{4}$ individuals $(0.0210 \%$ of the total of population) (see Figure 3$)$. Then, as is shown in Figure 2 and Figure 3, the percentage of symptomatic infectious decreases by increasing the percentage of isolation. As shown in Figure 2, the impact is not only on the decreasing of number of infectious, but also in the period of infection which is the more shorter in the scenario 3. Figure 4 shows that the number of recovered individuals increases rapidly in case of scenario 3 . In fact the maximum number of recovered in scenario 3 is $6.471 .10^{5}$ aginst $6.343 .10^{5}$ in scenario 2 and $6.001 .10^{5}$ in scenario 1 . Figures 2, 3 and 4 show the effectiveness of isolation of latent detectable and infectious symptomatic individuals in curtailing Ebola.

\section{Optimal control of the spread of the virus}

Epidemiological models have used optimal control techniques, most of which focus on HIV disease and tuberculosis (TB) [15, 16, 23, 24, 33, 34]. The optimal control efforts are carried out to limit the spread of the disease, and in some cases, to prevent the emergence of drug resistance. In this section, we formulate two strategies of optimal control problems subject to the $S E_{1} E_{2} I J R$ model (1), in order to derive the optimal isolation strategies. For each strategy, we study a specific objective in order to minimize not only the number of symptomatic infectious individuals or latent detectabe individuals but also the cost of the isolation program which includes the consumption for every individuals, the cost of organization and management. The isolation of symptomatic infectious and latent detectabe individuals has a great importance in countries that do not have the capacity to defend themselves against the virus.

We compare the result of each strategy with the simulation results studied in Section 3 for Liberia. The so called Strategy 1, which is described in Section 4.1, consists on the control of the virus by minimizing the symptomatic infectious and isolated individuals with the isolation cost, which includes the cost of consumption for every individuals, the cost of organization and management. Strategy 2 is an improvement of Strategy 1, is given in Section 4.2 and 
consists on the control of the virus by minimizing the symptomatic infectious, isolated and the latent detectable individuals with the isolation cost.

\subsection{Strategy 1}

In this section, we present a strategy of control of the virus by introducing into the model (1) a control $u(t)$ representing the isolation rate at time $t$. The control $u(t)$ is the fraction of symptomatic infectious individuals being isolated per unit of time. Then, the mathematical model with control is given by the following system of nonlinear differential equations:

$$
\begin{aligned}
& \frac{d S(t)}{d t}=\Lambda-\beta S(t)\left(\frac{I(t)+k J(t)}{N}\right)-\mu S(t), \\
& \frac{d E_{1}(t)}{d t}=\beta S(t)\left(\frac{I(t)+k J(t)}{N}\right)-\left(\sigma_{1}+\mu\right) E_{1}(t), \\
& \frac{d E_{2}(t)}{d t}=\sigma_{1} E_{1}(t)-\left(\sigma_{2}+\delta+\mu\right) E_{2}(t), \\
& \frac{d I(t)}{d t}=\sigma_{2} E_{2}(t)-(u(t)+\gamma+\mu) I(t), \\
& \frac{d J(t)}{d t}=u(t) I(t)+\delta E_{2}(t)-\left(\gamma_{r}+\mu\right) J(t), \\
& \frac{d R(t)}{d t}=\gamma I(t)+\gamma_{r} J(t)-\mu R(t) .
\end{aligned}
$$

The goal of the strategy is to reduce the symptomatic infectious individuals, the isolated individuals and the cost of isolation, which includes the cost of consumption for every individuals, the cost of organization, management and cooperation of the isolation program. Precisely, the optimal control problem consists of minimizing the objective functional

$$
J(u)=\int_{0}^{t_{\text {end }}}\left[A_{1} I(t)+A_{2} J(t)+\frac{C_{1}}{2} u^{2}(t)\right] d t,
$$

where $u(t)$ is the control variable, which represents the isolation rate at time $t, t_{\text {end }}$ denotes the duration of the isolation program, $A_{1}$ and $A_{2}$ are positive balancing cost factors due to size and importance of the parts of the objective function. The total cost includes the consumption for every individuals, the cost of organization, management and cooperation. Hence, the cost function should be nonlinear. In this paper, a quadratic function is implemented for measuring the control cost by reference to many papers in epidemic control $[35,36,37,38,39]$. 
In the quadratic term of (3), $C_{1}$ is a positive weight parameter associated with the control $u(t)$, and the square of the control variable reflects the severity of the side effects of the vaccination. One has $u \in \mathcal{U}_{a d}$, where

$$
\mathcal{U}_{\text {ad }}=\left\{u: u \text { is measurable, } 0 \leq u(t) \leq u_{\max }<\infty, t \in\left[0, t_{\text {end }}\right]\right\}
$$

is the admissible control set, with $u_{\max }=0.9$.

\subsection{Strategy 2}

In this strategy, we study the effect of isolation of latent detectable. Our idea is based on taking into acount the severity of the virus. In fact, let us recall that Ebola virus spreads through human-to-human transmission, not only by close and direct physical contact with infected bodily fluids, but also via exposure to objects or contaminated environment. The most infectious fluids are blood, feces, and vomit secretions. However, all body fluids have the capacity to transmit the virus. Here, we intend to control the propagation of the Ebola virus by using two control variables into the $S E_{1} E_{2} I J R$ model 1 . Then, the mathematical model with control is given by the following system of nonlinear differential equations:

$$
\begin{aligned}
& \frac{d S(t)}{d t}=\Lambda-\beta S(t)\left(\frac{I(t)+k J(t)}{N}\right)-\mu S(t), \\
& \frac{d E_{1}(t)}{d t}=\beta S(t)\left(\frac{I(t)+k J(t)}{N}\right)-\left(\sigma_{1}+\mu\right) E_{1}(t), \\
& \frac{d E_{2}(t)}{d t}=\sigma_{1} E_{1}(t)-\left(\sigma_{2}+u_{1}(t)+\mu\right) E_{2}(t), \\
& \frac{d I(t)}{d t}=\sigma_{2} E_{2}(t)-\left(u_{2}(t)+\gamma+\mu\right) I(t), \\
& \frac{d J(t)}{d t}=u_{2}(t) I(t)+u_{1}(t) E_{2}(t)-\left(\gamma_{r}+\mu\right) J(t), \\
& \frac{d R(t)}{d t}=\gamma I(t)+\gamma_{r} J(t)-\mu R(t) .
\end{aligned}
$$

The goal of the strategy is to reduce the latent detectable individuals, the symptomatic infectious individuals, the isolated individuals and the cost of isolation, which includes the cost of consumption for every individuals, the cost of organization, management and cooperation of the isolation program. Precisely, the optimal control problem consists of minimizing the objective functional

$$
J(u)=\int_{0}^{t_{\text {end }}}\left[B_{1} E_{2}(t)+B_{2} I(t)+B_{3} J(t)+\frac{C_{2}}{2} u_{1}^{2}(t)+\frac{C_{3}}{2} u_{2}^{2}(t)\right] d t,
$$


subject to the system 1 , where $u=\left(u_{1}, u_{2}\right)$, with $u_{1}$ representing the isolation control of latent detectable and $u_{2}$ the isolation control of symptomatic infectious individuals. The Lebesgue measurable control set is defined as

$$
\begin{gathered}
\mathcal{U}_{a d}:=\left\{u=\left(u_{1}, u_{2}\right): u \text { is measurable }, 0 \leq u_{1}(t), u_{2}(t) \leq u_{\max },\right. \\
\left.t \in\left[0, t_{\text {end }}\right]\right\}
\end{gathered}
$$

where $u_{\max }=0.9, C_{2}$ and $C_{3}$ are a positive weight parameters associated with the control $u_{1}(t)$ and $u_{2}(t)$. Here, we choose quadratic terms with respect to the controls in order to describe the nonlinear behaviour of the cost of implementing the isolation program. The parameters $B_{1}, B_{2}$ and $B_{3}$ are positive balancing cost factors due to size and importance of the parts of the objective function. In the objective functional, the terms $B_{1} u_{1}^{2} / 2$ and $B_{2} u_{2}^{2} / 2$ represents the cost associated with the isolation program which includes the consumption for every individuals, the cost of organization, management and cooperation.

\subsection{Discussion}

In this section we compare between the two strategies and the case without control, and we discuss the obtained results. In order to compare the optimal control study of strategy 1 and strategy 2 with the numerical solution of the model 1 without control, we use here the same parameters, and the same initial values details in Tables 1, 2 and 3 for the initial number of susceptible, latent undetectable, latent detectable, symptomatic infectious, isolated and recovered populations. In Strategy $1, t_{\text {end }}=120$ days, $C_{1}=200, A_{1}=1$ and $A_{2}=1$. In Strategy 2, the values of the parameters of the objective function are given by: $C_{2}=200, C_{3}=200, B_{1}=1, B_{2}=1, B_{3}=1$, where $t_{\text {end }}=120$ days.

The same parameters defined in Section 3 were used in the control study of study. For the numerical solutions of the optimal control problems, we have used the ACADO solver [40], which is based on a multiple shooting method, including automatic differentiation and based ultimately on the semidirect multiple shooting algorithm of Bock and Pitt [41]. The ACADO solver comes as a selfcontained public domain software environment, written in $\mathrm{C}++$, for automatic control and dynamic optimization.

Figures 5, 6, 7 and 8 show respectively the time-dependent curves of latent detectable, symptomatic infectious, isolated and recovered individuals in case of control with Strategy 1, control with Strategy 2 and the case without control, for the study of the population of Liberia. Figure 5 shows that the number of latent detectable $E_{2}$ decreases more rapidly in case of optimal control under Strategy 2 than in case of control with Strategy 1 and the case without con- 
trol, during the isolation campaign. In Figure 6, the time-dependent curve of symptomatic infectious individuals shows that there is no peak of the curve of infectious individuals in case of control with Strategy 1 and Strategy 2, against the case without control in which an important peak appears. The same curves show that the period of infection is shorter in case of control with Strategy 2 than in case of control with strategy 1 which is shorter than the case without control. This shows the efficiency of isolation control with Strategy 1 and Strategy 2 in controlling the virus. Figure 7 presents the time-dependent curve of isolated individuals of Liberia. Figure 8 show that the number of recovered individuals increases more rapidly in case of control with Strategy 2. The number of recovered increases more rapidly in case of control with Strategy 1 than the case without control.

Figure 9 gives a representation of the time dependent optimal control variable $u(t)$ for Strategy 1. Figure 10 presents the time dependent optimal controls $u_{1}(t)$ and $u_{2}(t)$. In the two strategies, control variables stay at the upper bound during the beginning of the isolation program and start to decrease after. By comparing between the two optimal controls $u_{1}(t)$ and $u_{2}(t)$, in Strategy 2, we see that the control variable $u_{2}$ takes longer time at the upper bound, than the control $u_{1}$.

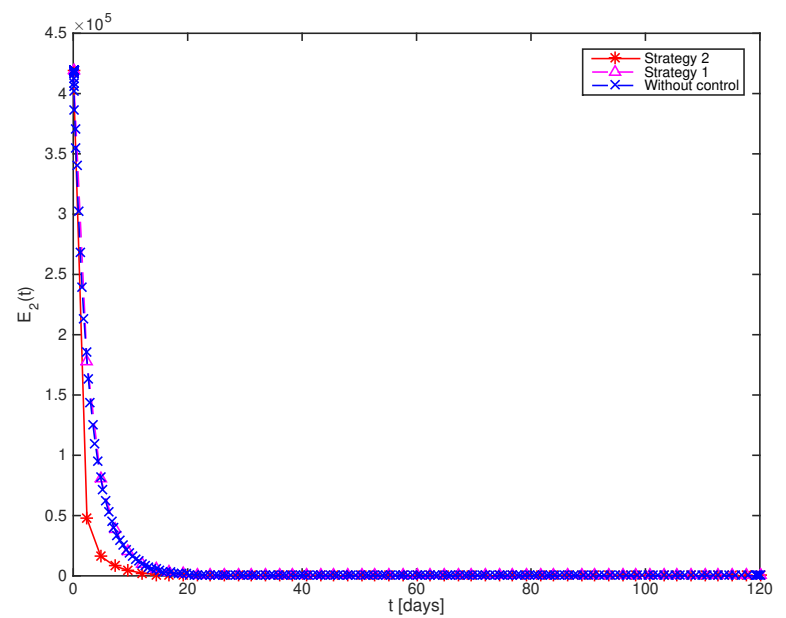

Figure 5: Evolution of individuals in compartment $E_{2}(t)$ in case of control with Strategy 1, Strategy 2 and the case without control. 


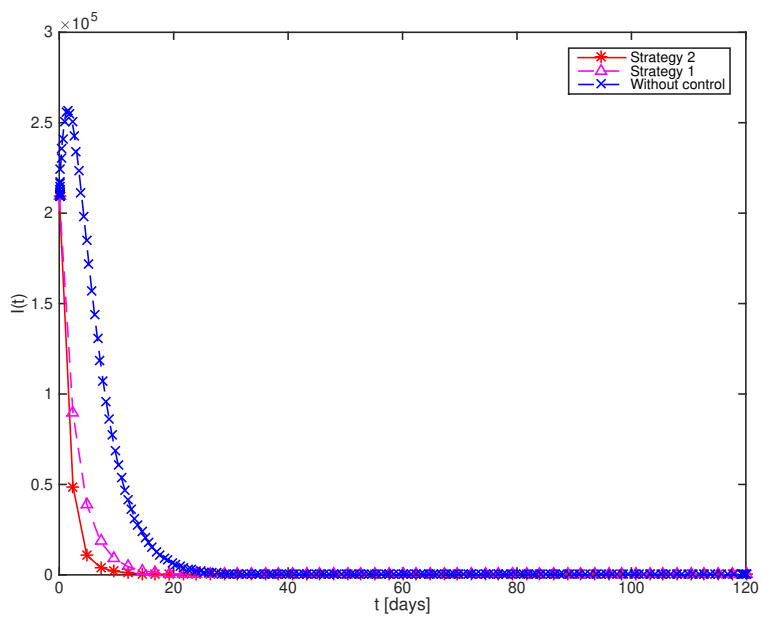

Figure 6: Evolution of individuals in compartment $I(t)$ in case of control with Strategy 1, Strategy 2 and the case without control.

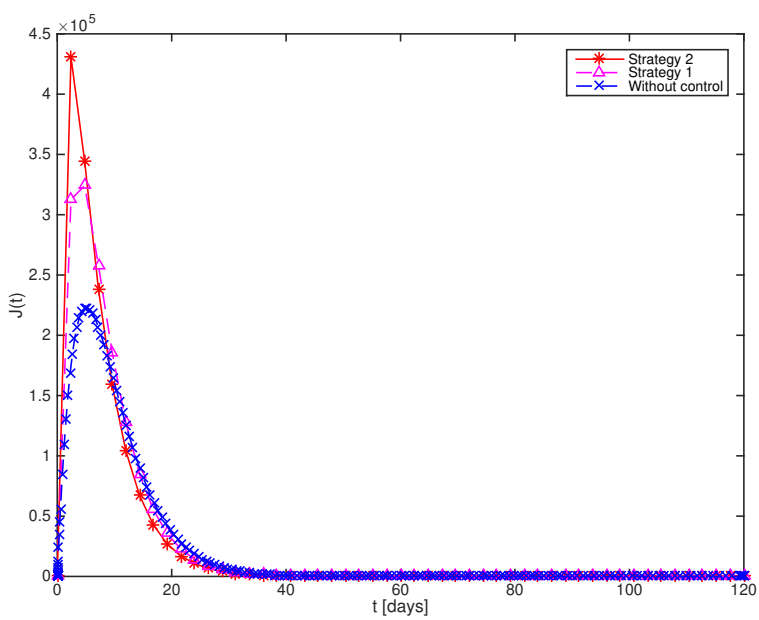

Figure 7: Evolution of individuals in compartment $J(t)$ in case of control with Strategy 1, Strategy 2 and the case without control.

\section{Conclusions}

We investigated a $S E_{1} E_{2} I J R$ model which describes the 2015 Ebola virus outbreak occurred in Liberia taking into account demographic effects, latent unde- 


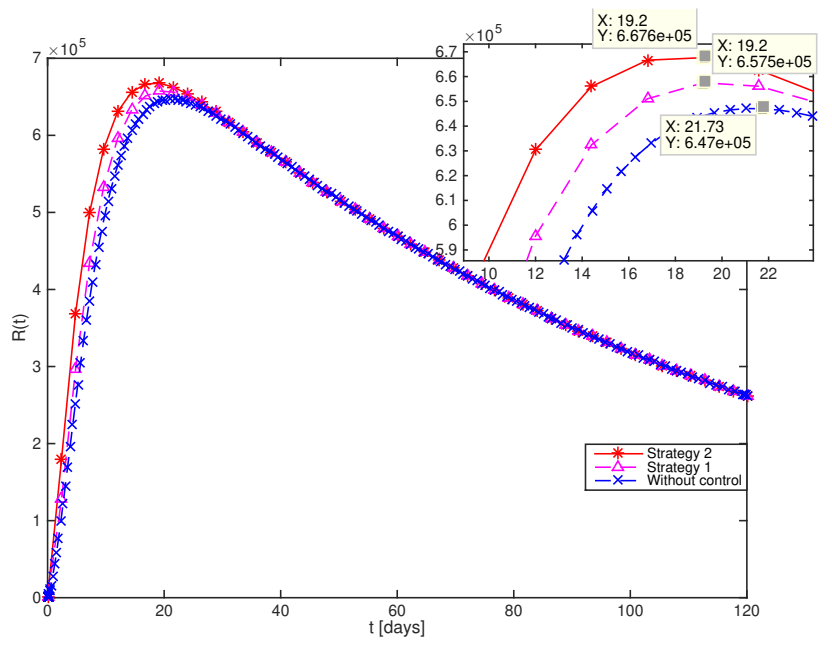

Figure 8: Evolution of individuals in compartment $R(t)$ in case of control with Strategy 1, Strategy 2 and the case without control.

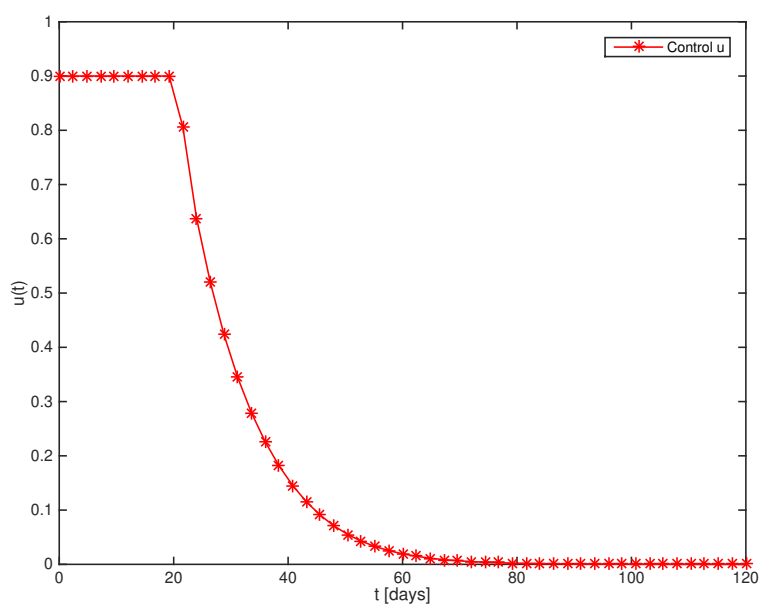

Figure 9: The optimal control $u$ for Strategy 1.

tectable and latent detectable compartments with isolation of infectious individuals. We resolved numerically the model in order to assess the population-level impact of isolation of symptomatic individuals. It has been shown that isolating latent detectable and infectious individuals is the most effective in curtailing 


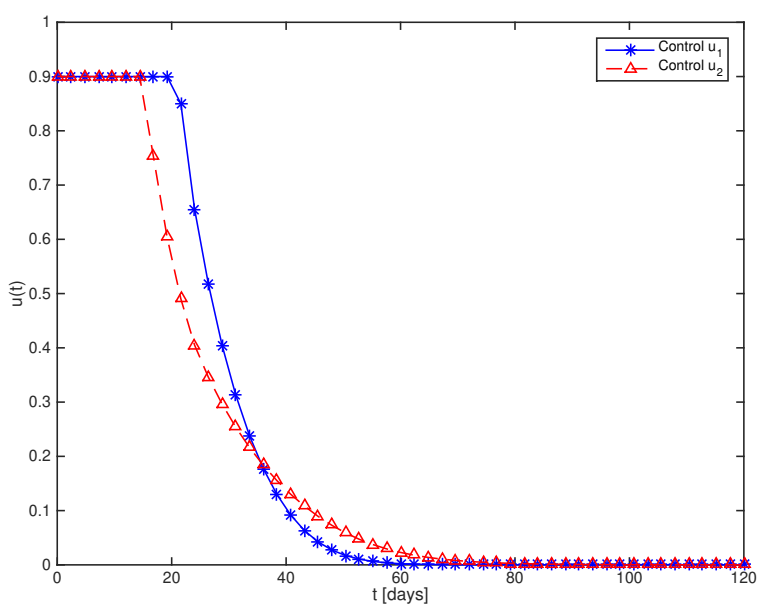

Figure 10: The optimal control variables $u_{1}$ and $u_{2}$ for Strategy 2 .

the virus.

\section{References}

[1] S. Shrivastava, P. Shrivastava, World Health Organization releases the list of blueprint priority diseases, Journal of the Scientific Society, 45 (2018), 49-49.

[2] M. Barry, F.A. Traoré, F.B. Sako, D.O. Kpamy, E.I. Bah, M.Poncin, S. Keita, M. Cisse, A. Touré, Ebola outbreak in Conakry, Guinea: epidemiological, clinical, and outcome features, Medecine et maladies infectieuses, 44 (2014), 491-494.

[3] E.K. Chapnick, Ebola Myths and Facts for Dummies, John Wiley \& Sons (2014).

[4] A.J. Lewnard, M.L.N. Mbah, J.A. Alfaro-Murillo, L.F. Altice, L. Bawo, G.T. Nyenswah, P.A. Galvani, Dynamics and control of Ebola virus transmission in Montserrado, Liberia: a mathematical modelling analysis, The Lancet Infectious Diseases, 14 (2014), 1189-1195.

[5] C.T. Smith, H. Babcock, Ebola and Marburg Viruses, Infobase Publishing (2010). 
[6] L. Borio, T. Inglesby, C.J. Peters, A.L. Schmaljohn, J.M. Hughes, P.B. Jahrling, T. Ksiazek, K.M. Johnson, A. Meyerhoff, T. O'Toole, Hemorrhagic fever viruses as biological weapons: medical and public health management, Jama, 287 (2002), 2391-2405.

[7] S.F. Dowell, R. Mukunu, T.G. Ksiazek, A.S. Khan, P. E. Rollin, E. Pierre, C.J. Peters, Transmission of Ebola hemorrhagic fever: a study of risk factors in family members, Kikwit, Democratic Republic of the Congo, 1995, The Journal of Infectious Diseases, 179 (1999), 87-91.

[8] C.T. Smith, Ebola, Infobase Publishing (2009).

[9] J. Alton, The Ebola Survival Handbook: An MD Tells You What You Need to Know Now to Stay Safe, Simon and Schuster (2014).

[10] J. Legrand, R.F. Grais, P.Y. Boelle, A.J. Valleron, A. Flahault, Understanding the dynamics of Ebola epidemics, Epidemiology \& Infection, 135 (2007), 610-621.

[11] S.I. Okware, F.G. Omaswa, S. Zaramba,A. Opio, J.J. Lutwama, J. Kamugisha, E.B. Rwaguma, P. Kagwa, M. Lamunu, An outbreak of Ebola in Uganda, Tropical Medicine \& International Health, 7 (2002), 1068-1075.

[12] K. Bibby, L.W. Casson, E. Stachler, C.N. Haas, Ebola virus persistence in the environment: state of the knowledge and research needs, Environmental Science \& Technology Letters, 2 (2015), 2-6.

[13] A. Rachah, D.F.M. Torres, Mathematical modelling, simulation, and optimal control of the 2014 Ebola outbreak in West Africa, Discrete Dynamics in Nature and Society, 2015 (2015).

[14] A. Rachah, D.F.M. Torres, Optimal control strategies for the spread of Ebola in West Africa, Journal of Mathematical Analysis, 7 (2016), 102-114.

[15] S. Gao, Z. Teng, J.J. Nieto, A. Torres, Analysis of an SIR epidemic model with pulse vaccination and distributed time delay, BioMed Research International, 2007 (2007).

[16] C.M. James-Berry, Vaccine control of avian influenza H5N1 in poultry: need for a positive marker, Journal of Vaccines \& Vaccination, 4 (2013), 168-178. 
[17] E. Jung, S. Iwami, Y. Takeuchi, T. Jo, Optimal control strategy for prevention of avian influenza pandemic, Journal of theoretical biology, $\mathbf{2 6 0}$ (2009), 220-229.

[18] J.M. Tchuenche, S.A. Khamis, F.B. Agusto, S.C. Mpeshe, Optimal control and sensitivity analysis of an influenza model with treatment and vaccination, Acta Biotheoretica, 59 (2011), 1-28.

[19] A. Rachah, D.F.M. Torres, Predicting and controlling the Ebola infection, Mathematical Methods in the Applied Sciences, 40 (2017), 6155-6164.

[20] O. Diekmann, H. Heesterbeek, T. Britton, Mathematical Tools for Understanding Infectious Disease Dynamics, Princeton University Press, Oxford (2012).

[21] M. Kretzschmar, S. Van den Hof, J. Wallinga, J. Van Wijngaarden, Ring vaccination and smallpox control, Emerging Infectious Diseases, 10 (2004), $832-841$.

[22] J. Longini, M. Ira E. Ackerman, L.R. Elveback, An optimization model for influenza A epidemics, Mathematical Biosciences, 38 (1978), 141-157.

[23] H.S. Rodrigues, M.T. Monteiro, D.F.M. Torres, Dynamics of dengue epidemics when using optimal control, Mathematical and Computer Modelling, 52 (2010), 1667-1673.

[24] H.S. Rodrigues, M.T. Monteiro, D.F.M. Torres, Vaccination models and optimal control strategies to dengue, Mathematical Biosciences, 247 (2014), 1-12.

[25] M. Eichner, S.F. Dowell, N. Firese, Incubation period of Ebola hemorrhagic virus subtype Zaire, Osong Public Health and Research Perspectives, 2 (2011), 3-7.

[26] E.M. Leroy, S. Baize, V.E. Volchkov, S.P. Fisher-Hoch, M.C. GeorgesCourbot, J. Lansoud-Soukate, M. Capron, P. Debre, A.J. Georges, J.B. McCormick, Human asymptomatic Ebola infection and strong inflammatory response, The Lancet, 355 (2000), 2210-2215.

[27] WHO Ebola Response Team, Ebola virus disease in West Africa - the first 9 months of the epidemic and forward projectionse, New England Journal of Medicine, 371 (2014), 1481-1495. 
[28] G. Chowell, H. Nishiura, Transmission dynamics and control of Ebola virus disease (EVD): a review, BMC Medicine, 12 (2014).

[29] P.E. Lekone, B.F. Finkenstädt, Statistical inference in a stochastic epidemic SEIR model with control intervention: Ebola as a case study, Biometrics, 62 (2006), 1170-1177.

[30] A. Khan, M. Naveed, M. Dur-e-Ahmad, M. Imran, Estimating the basic reproductive ratio for the Ebola outbreak in Liberia and Sierra Leoney, Infectious Diseases of Poverty, 4 (2015).

[31] http://www.statistiques-mondiales.com/liberia.html, 2015.

[32] H.R. Thieme, Mathematics in Population Biology, In: Princeton Ser. in Theoretical and Computational Biology, United States of America (2003).

[33] P. Rodrigues, J. Cristiana D.F.M. Torres, Cost-effectiveness analysis of optimal control measures for tuberculosis, Bulletin of Mathematical Biology, 76 (2014), 2627-2645.

[34] S.J. Cristiana, D.F.M. Torres, Optimal control for a tuberculosis model with reinfection and post-exposure interventions, Mathematical Biosciences, 244 (2013), 154-164.

[35] M.A.L. Caetano, T. Yoneyama, Optimal and sub-optimal control in Dengue epidemics, Optimal Ccontrol Applications and Methods, 22 (2001), 63-73.

[36] J.F. De Souza, T. Yoneyama, Optimization of investment policies in the control of mosquito-borne diseases, In: American Control Conference, IEEE (1992), 681-682.

[37] E. Jung, S. Lenhart, Z. Feng, Optimal control of treatments in a twostrain tuberculosis model, Discrete \& Continuous Dynamical Systems-B, 2 (2002), 473-482.

[38] D. Kirschner, Denise and S. Lenhart, S. Serbin, Optimal control of the chemotherapy of HIV, Journal of Mathematical Biology, 35 (1997), 154164.

[39] J.F. De Souza, T. Yoneyama, Optimization of the investment in educational campaigns in the control of insect transmitted diseases, In: IEEE International Conference on Control and Applications, IEEE (1994), 16891694. 
[40] D. Ariens, B. Houska, H.J. Ferreau, ACADO Toolkit User's Manual, Toolkit for Automatic Control and Dynamic Optimization, (2010).

[41] H.G. Bock, K.J. Plitt, A multiple shooting algorithm for direct solution of optimal control problems, IFAC Proceedings Volumes, 17 (1984), 16031608. 